\title{
Multi-modal Imaging of Cell-Biomaterial Interactions Under Dynamic Chemical and Mechanical Environments
}

\section{Wei Tan}

Departments of Mechanical Engineering, Bioengineering and Pediatrics, University of Colorado, 12700 E $19^{\text {th }}$ Ave, RCII Room 6112, Aurora, CO 80045

Studying cell-biomaterial interactions and cell activities in three-dimensional (3-D) biomaterials constitutes a critical research component to enhance our fundamental understanding of tissue engineering process. Such understanding can guide us to develop or improve a process. Recently, a great deal of interest has arisen in designing and controlling dynamic biochemical and biomechanical environments to produce engineered tissues. Though significant advancement has been made in this area, lack of the information on how cell-biomaterial interactions and cell activities in 3-D biomaterials respond to the chemical and mechanical environments prevents the researchers from systematically analyzing the impacts of these environmental factors on cells and biomaterials and optimizing the dynamic processes. As a result, when conditioning a tissue engineering process, somewhat random approaches are often taken towards defining the spatiotemporal distribution of chemical $/ \mathrm{molecular}$ environments or the patterns of mechanical stimuli. A multi-modal, non-invasive, 3-D imaging platform is thus developed to analyze cell dynamics and cell-biomaterial interactions in response to a variety of environments. This platform combines coherent and incoherent imaging for spatial and temporal visualization of cells, biomaterials and molecules. This approach harnesses a combined microscopy technology of optical coherence microscopy (OCT) and multiphoton microscopy (MPM) together with green fluorescent protein (GFP) technology. Compared with conventional invasive, destructive imaging techniques such as histology and SEM, OCT and MPM provide noninvasive, time-lapse imaging which reveals complementary characteristics of cells and tissues such as tissue architecture and development stages, cell distribution and biology, and cell-biomaterial interaction. The elimination of specimen fixation and extensive processing reduces the possibility of structural artifacts and facilitates repeated observations within a single sample over time, and in response to various internal and external chemical and mechanical stimuli. This can greatly benefit the investigation of tissue engineering by pushing forward scientific understanding of 3-D cell culture in response to dynamic engineering environments.

The 3-D visualization of an engineered tissue made of fibroblasts and porous chitosan scaffold was achieved by volume-rendering a series of cross-sectional OCT or MPM images (Fig. 1). By assembling sequential crosssectional OCT images with $20 \mu \mathrm{m}$ interval in space, we obtained 3-D structural information about the engineered tissues longitudinally over time. The engineered tissue clearly exhibited a porous microarchitecture. Using transfected GFP-vinculin, we also demonstrated the changes in cell morphology, matrix production, cell-scaffold adhesion (GFP-vinculin) and 3-D engineered tissue over the long-term culture period. Vinculin is an abundant cytoskeletal protein found in integrin-mediated focal adhesions and also in cadherin-mediated cell-cell adherens junctions. The dye separation algorithm separates the emitted GFP fluorescence from the background chitosan scaffold autofluorescence and the reflected signals from cell-produced extracellular matrix.

Using the multi-modal imaging platform, we also obtained complementary structural and functional information of dynamics of engineered tissues (including cells, scaffolds and cell-scaffold interactions) under mechanicallystimulated culture conditions. Representative 3-D reconstructed images of the cell-chitosan constructs before and after $24 \mathrm{hr}$ of cyclic sinusoidal stretching at a frequency of $1 \mathrm{~Hz}$ were shown in Fig 2 . The figures demonstrate cell responses in the 3-D constructs to mechanical stretching, and more importantly, demonstrate the underlying mechanistic mechanism of cell responses by showing the structural changes of the scaffold due to mechanical deformation over time.

Cell migration and cell death are both important cellular responses to biochemical environments such as cytokines and drugs. But currently it is difficult to control these dynamic processes for cells cultured in a 3-D biomaterial construct. Multimodal imaging with OCT and MPM allow one to analyze the cell migration in a 3-D matrix construct towards environmental chemoattractants (i.e. MCP-1) or to analyze the cell death process in response to environmental toxicants or drugs (Fig 3). The figures demonstrate the positional changes or optical scattering changes of the cells in a 3-D tissue model over time. The migration direction and speed calculated from both 
types of images were comparable. Cell positions at each specific time-point were labeled with a specific color and combined into composite images in OCT images. We thus tracked the paths traveled by individual cells and determine the distribution of phenomenological parameters. Additionally, using OCT to dynamically monitor the changes in cell optical scattering properties and 3-D cell morphology in a tissue model, we not only detected the different stages of cell death, necrosis or apoptosis, but also differentiated these two mechanisms in response to different environmental biochemicals such as toxicant (i.e. bleach) and apoptosis-inducing drugs (i.e. camptothecin and etoposide, or $\mathrm{C}+\mathrm{E}$ ).
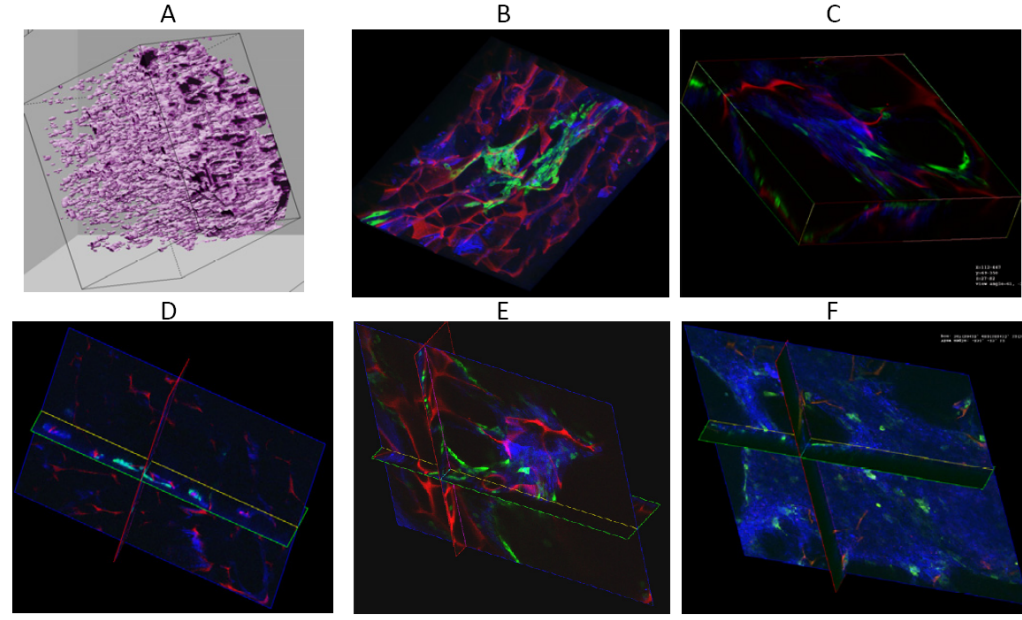

Figure 1. Three-dimensional reconstructed OCT (A) and MPM (B-F) images of engineered tissue. The 3-D rendered volumes are viewed at a rotational angle (B), with a cubic structure (C) or with cross-sectional planes to compare changes in cell-biomaterial interactions after cultured for 1 day (D), 3 days (E), and 6 days(F).
A

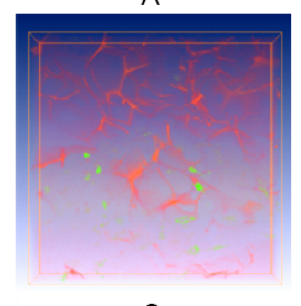

C

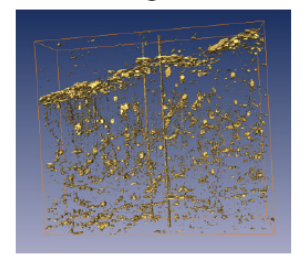

B

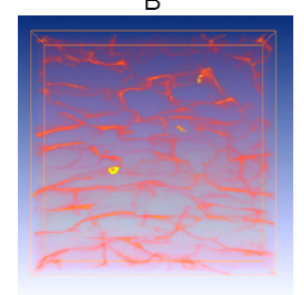

D

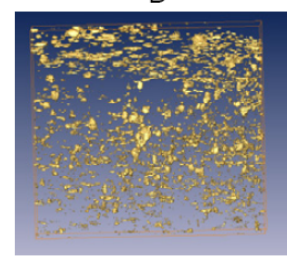

Figure 2. Three-dimensional reconstructed MPM (A, B) and OCT (C, D) images of engineered tissues before $(\mathrm{A}, \mathrm{C})$ and after $(\mathrm{B}, \mathrm{D})$ cyclic mechanical stretching of the tissue constructs

A

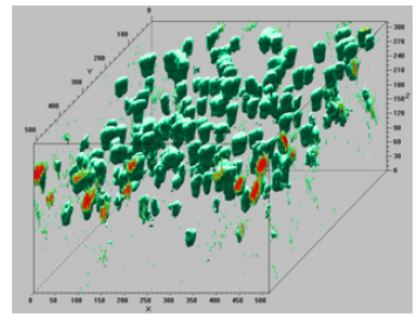

E

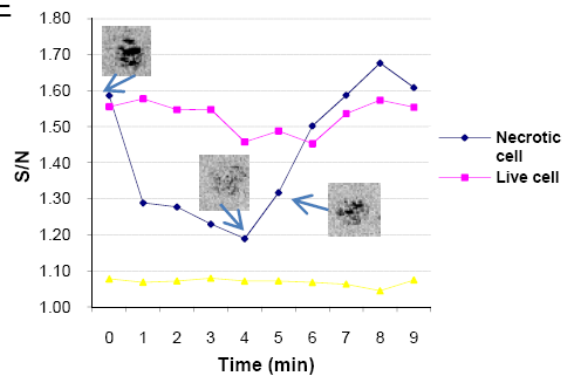

B

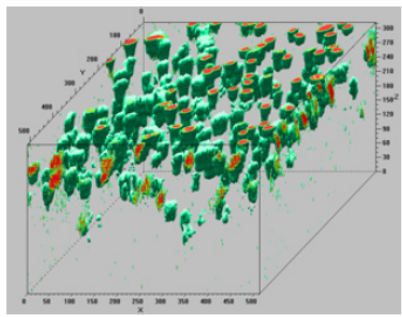

C

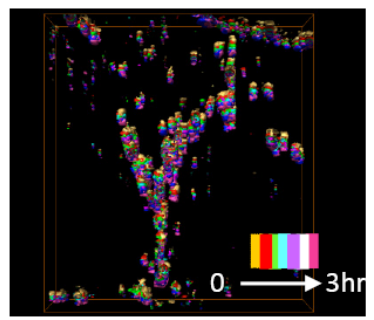

$\mathrm{D}$

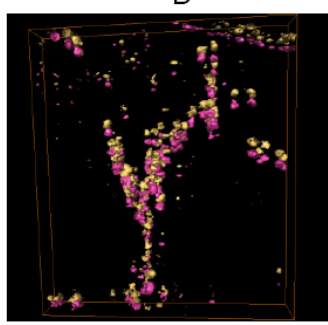

$\mathrm{F}$

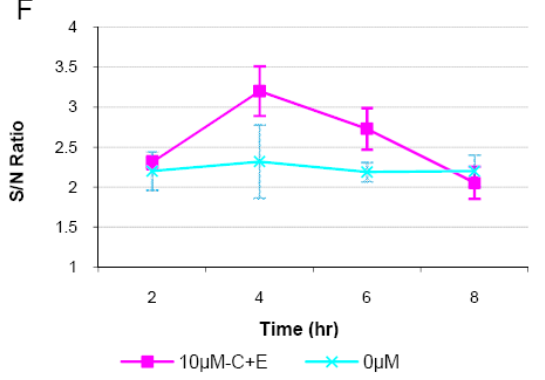

G
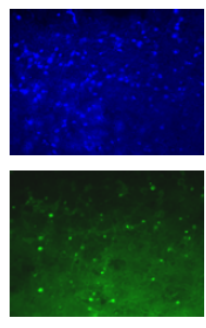

Figure 3. Three-dimensional reconstructed MPM (A-B) and OCT (C-D) images of engineered tissues showing cell migration in the 3-D biomaterial constructs in response to a chemoattractant. Figures $\mathrm{C}$ and $\mathrm{D}$ show the overlaid 3-D OCT images acquired at continuous points of time (every 30min). (E-F) OCT results demonstrate the optical properties of the cells that show different dynamic changes in response to toxicants (undergoing necrotic death) vs drugs (undergoing apoptotic death). (G) Fluorescent images of the tissue blocks stained with Fluorescien-FragEL DNA fragmentation kit after drug treatment. 